\title{
Le Père Jean-Baptiste de La Brosse, professeur, linguiste et ethnographe chez les Montagnais du Saguenay (1766-1782)
}

\section{Léo-Paul Hébert}

Volume 55, 1988

Cent cinquante ans d'histoire religieuse au Saguenay-Lac-Saint-Jean (1838-1988)

URI : https://id.erudit.org/iderudit/1006944ar

DOI : https://doi.org/10.7202/1006944ar

Aller au sommaire du numéro

Éditeur(s)

Les Éditions Historia Ecclesiæ Catholicæ Canadensis Inc.

ISSN

0318-6172 (imprimé)

1927-7067 (numérique)

Découvrir la revue

Citer cet article

Hébert, L.-P. (1988). Le Père Jean-Baptiste de La Brosse, professeur, linguiste et ethnographe chez les Montagnais du Saguenay (1766-1782). Sessions d'étude Société canadienne d'histoire de l'Église catholique, 55, 7-39.

https://doi.org/10.7202/1006944ar
Résumé de l'article

De 1766 à 1782, le P. de la Brosse dessert les Montagnais du Saguenay; en 1767, il fait imprimer 2000 exemplaires du Nehiro-Iriniu (catéchisme et livre de prières) et 3000 abécédaires; grâce à ces instruments, il montre à lire et à écrire aux Montagnais; ainsi ils pourront plus facilement apprendre leur catéchisme et leurs prières, et surtout se transmettre leurs connaissances les uns les autres, et de père en fils.

Le P. de La Brosse fut célèbre chez les Montagnais et devint homme de légende : un jour il aurait arrêté un feu de forêt (voir bronze du sculpteur Alfred Laliberté) et à son décès les cloches de la chapelle de Tadoussac auraient sonné d'elles-mêmes pour annoncer sa mort? Où finit l'histoire? Où commence la légende?
Tous droits réservés @ Ces Éditions Historia Ecclesiæ Catholicæ Canadensis Inc., 1988
Ce document est protégé par la loi sur le droit d'auteur. L'utilisation des services d'Érudit (y compris la reproduction) est assujettie à sa politique d'utilisation que vous pouvez consulter en ligne. 


\title{
Le Père Jean-Baptiste de La Brosse, professeur, linguiste et ethnographe chez les Montagnais du Saguenay $(\mathbf{1 7 6 6 - 1 7 8 2})^{1}$
}

\author{
Léo-Paul HÉBERT \\ Département des Sciences Humaines \\ Cégep Joliette-De Lanaudière
}

\section{RÉSUMÉ}

De 1766 à 1782, le P. de la Brosse dessert les Montagnais du Saguenay; en 1767, il fait imprimer 2000 exemplaires du Nehiro-Iriniu (catéchisme et livre de prières) et 3000 abécédaires; grâce à ces instruments, il montre à lire et à écrire aux Montagnais; ainsi ils pourront plus facilement-apprendre leur catéchisme et leurs prières, et surtout se transmettre leurs connaissances les uns les autres, et de père en fils.

Le P. de La Brosse fut célèbre chez les Montagnais et devint homme de légende: un jour il aurait arrêté un feu de forêt (voir bronze du sculpteur Alfred Laliberté) et à son décès les cloches de la chapelle de Tadoussac auraient sonné d' elles-mêmes pour annoncer sa mort? Où finit l' histoire? Où commence la légende?

\section{Introduction}

Le P. Jean-Baptiste de La Brosse est plus célèbre par la légende que par l'histoire: à Tadoussac et dans la région du Bas-du-Fleuve, il aurait accompli des prodiges. À sa mort, dont il aurait prédit l'heure exacte, les cloches de la chapelle de Tadoussac et de toutes les autres églises qu'il desservait auraient sonné d'elles-mêmes. On dit aussi qu'il aurait arrêté un

1 La présente communication est tirée en partie des publications suivantes: Histoire ou légende? Jean-Baptiste de La Brosse, Montréal, Bellarmin, 1984, 546 p. «Jean-Baptiste de La Brosse», Dictionnaire biographique du Canada, tome IV, p. 457-460. Le troisième registre de Tadoussac - Miscellaneorum Liber, [Édition critique], Montréal, Les Presses de l'Université du Québec (Coll. «Tekouerimat» 3), 1976, XXII et 340 p. Le quatrième registre de Tadoussac [Édition critique], Sillery, Les Presses de l'Université du Québec (Coll. «Tekouerimat» 8), 1982, XXXIX et 144 p. 
feu de forêt. Dans un de ses bronzes, le sculpteur Alfred Laliberté l'a choisi comme type du "prêtre conjurant le feu». Mais où finit l'histoire et où commence la légende?

Jean-Baptiste de La Brosse naquit le 30 avril 1724 dans le hameau de Magnac, paroisse de Jauldes (Charente). Il était le septième et dernier enfant de Louise Duboiscuvier et de Jean-Baptiste de La Brosse, sieur de la Chabrouillère et de Magnac.

Après ses études secondaires au collège des Jésuites d'Angoulême, il fait son noviciat chez les Jésuites de Bordeaux de 1742 à 1744, puis selon la coutume, il enseigne dans divers collèges. De 1749 à 1754, il fait une troisième année de philosophie et ses quatre ans de théologie. Il avait été ordonné prêtre en 1753.

Arrivé au Canada en 1754, il est d'abord affecté au Collège des Jésuites de Québec. En 1755-1756, on le retrouve en Acadie, où il accompagne les Acadiens dans leur fuite à travers les bois.

De retour à Qućbcc, il enseigne la philosophie au Collège des Jésuites ${ }^{2}$ jusqu'en 1758, alors qu'il devient missionnaire des Abénaquis de Saint-François (Odanak). Avec eux, il participe au siège de Québec, est fait prisonnier par les Anglais le 9 juillet 1759 et libéré le lendemain. Au cours des années 1758-1759, il a composé un Dictionnaire abénaquis.

En 1760-1761, le P. de La Brosse dessert la paroisse de Terrebonne; puis il devient curé de Saint-Henri de Mascouche de 1761-1766. C'est alors que Mgr Jean-Olivier Briand le nomme missionnaire des Montagnais du Saguenay. Il avait 42 ans.

Dans ses Annales, il signale son arrivée à Tadoussac avec beaucoup de solennité:

L'Année du Seigneur mil sept cent soixante-six, le onze juillet, à six heures du soir, moi, Jean-Baptiste de La Brosse, missionnaire, originaire de l'Angoumois, âgé de quarante-deux ans, deux mois et dix jours, j'arrivai à Tadoussac;

Il y avait vingt-cinq ans et neuf mois que j'étais entré dans la Compagnie de Jésus; treize ans, trois mois et trois jours que j'avais été promu au sacerdoce; onze ans, dix mois et cinq jours que j'avais accosté sur les rives canadiennes; cinq ans, dix mois et deux jours que le pays avait capitulé et était passé sous la domination anglaise; alors que Messire Guy Carleton était gouverneur, que Monseigneur Jean-Olivier Briand était évêque et que le Révérend Père Augustin de Glapion

${ }^{2}$ Les archives du Séminaire de Québec conservent son cours de philosophie transcrit par André Couilard: Cursus philosophicus a patre Labrosse in Collegio Quebecensi anno 1757-Philosophia, pars 1a, logica. 
était recteur et supérieur général des missions de la Compagnie de Jésus, j'entrepris le ministère des missions montagnaises ${ }^{3}$.

\section{Sommaire:}

1. Le milieu

2. Le missionnaire et le professeur

3. Le linguiste

4. L'ethnolgraphe

5. Résultats et prolongements de l'œuvre

Quel était l'état du milieu montagnais à l'arrivée du P. de La Brosse en 1766 ? Le P. de La Brosse fut-il plus missionnaire que professeur? quelle est l'importance de son œuvre comme linguiste et ethnographe? Enfin quels furent les résultats de son œuvre d'alphabétisation chez les Montagnais?

\section{Le milieu}

En juillet 1766, Mgr Briand vient de revenir d'Europe, où il a réussi à se faire ordonner évêque. En 1764, le clergé comptait 133 prêtres pour une population d'environ 70000 habitants. Il reste encore 19 jésuites au Canada. La Compagnie de Jésus sera supprimée par le Pape Clément XIV en 1773, mais au Canada on gardera la chose secrète; le gouvernement anglais a interdit aux Jésuites de se recruter, mais ils pourront jouir de leurs biens jusqu'à la mort du P. Casot en 1800.

Le P. de La Brosse succède au P. Claude-Godefroy Coquart décédé à Chicoutimi en 1765.

LE DOMAINE DU ROI. - En 1766, le nouveau champ d'apostolat du P. de La Brosse est le Domaine du Roi. C'est un territoire immense réservé à la traite des fourrures. Seuls les commerçants et les missionnaires $y$ ont accès.

Sur la rive nord du Saint-Laurent, le domaine s'étend de l'île aux Coudres à la rivière Moisie.

Au sud, la limite était une ligne allant de la pointe nord-est de l'île aux Coudres à la source de la rivière Métabetchouan.

Au nord, le Domaine s'étendait indéfiniment ${ }^{4}$.

3 Annales, nos 1-2. Les Annales Missionis ab Anno 1766 du P. de La Brosse sont inscrites dans le Troisième registre de Tadoussac, fol. 87v-90r, Archives de l'Archevêché de Québec. Les numéros renvoient à notre édition et à notre traduction française des Annales, parues dans Ilistoire ou légende? Jean-Baptiste de La Brosse, Montréal, Bellarmin, 1984, p. 176-193.

4 Victor Tremblay, Histoire du Saguenay depuis les origines jusqu' à 1870, Publication de La Société historique du Saguenay, ${ }^{\circ} 21,1968$, p. 194. 
LES MONTAGNAIS. - Les différents groupes du Saguenay sont désignés sous les noms de Tadoussacciens, Papinachois, Betsiamites, Oumamioueks, Chicoutimiens, Porcs-Épics, Nekoubistes, Mistassins, Gens «des terres» (de l'intérieur).

MODE DE VIE. - Les Montagnais vivaient principalement de chasse et de pêche; mais depuis l'arrivée des Européens, leur mode de vie a été profondément transformé. Toute leur existence dépend maintenant de leur chasse et des maigres profits qu'ils peuvent tirer des fourrures. Après l'hiver passé dans la forêt, ils reviennent de l'intéricuir des terres et se rassemblent aux divers postes de traite, pour négocier leurs fourrures et rencontrer le missionnaire.

LE CHRISTIANISME CHEZ LES MONTAGNAIS. - En 1766, la majorité des Montagnais est baptisée et on compte chez eux un grand nombre de chrétiens de vieille souche. De plus il y a des traditions bien établies et des coutumes respectées par les missionnaires précédents, par le P. Coquart entre autres.

LA POPUlation MONTAgNaise. - Au temps du P. de La Brosse, leur nombre devait se situer entre 5000 et 6000 habitants $^{5}$.

Le recensement de Peter Stuart de 1778 donne 3500 âmes6.

LA FERME DU DOMAINE DU ROI. - Depuis 1762, la traite du Domaine du Roi est affermée à une société formée de John Gray et de Thomas Dunn, marchands de Québec, à qui vont se joindre William Grant et Peter Stuart. Jusqu'en 1786, cette compagnie aura l'exclusivité de la traite des fourrures du Domaine du Roi.

Les sites des postes de traite ont varié. Au temps de La Brosse, ce sont: Tadoussac, les Îlets de Jérémie, Sept-Îles, Chicoutimi et quelques postes éphémères: Portneuf, la Pointe de Betsiamite et la Pointe des Monts.

FONCTIONNEMENT DES POSTES. - Le site du poste comprend habituellement les magasins ou entrepôts, la forge, la boulangerie, le logement du commis, la chapelle et parfois la maison du missionnaire.

Le premier personnage du poste est le commis, représentant immédiat des Bourgeois de Québec, assisté d'un personnel plus ou moins nombreux, selon l'importance du poste et le temps de l'année: assistant-commis, forgeron, ou armurier, tonnelier et les engagés de divers métiers. Le poste de Tadoussac compte 16 personnes en $1783^{7}$.

\footnotetext{
5 V. Tremblay, Histoire du Saguenay, 1968, p. 98.

6 Journaux de la Chambre d'Assemblée du Bas-Canada, 1823-1824, vol. 33, app. (R).

7 La Brosse au grand Vicaire Gravé, Tadoussac, 21 avril 1780 (Arch. de l'archevêché de Rimouski).
} 
Certains commis et engagés passent de nombreuses années dans les Postes: Charles Taché y résidera 30 ans.

Pendant l'hiver, le personnel diminue, mais le poste n'est pas complètement désert. Il reste toujours quelques familles amérindiennes, comprenant des personnes plus âgées, des enfants; on fera la chasse dans les environs du poste.

Voilà la «province8», selon l'expression de Mgr Briand, que La Brosse aura à desservir de 1766 à 1782.

Aux missions montagnaises, La Brosse devra bientôt ajouter d'autres activités. Durant l'hiver 1769-1770, il sera curé de l'île d'Orléans et à partir de 1770, Mgr Briand lui confie le service de la rive sud du SaintLaurent (Cacouna, l'Isle-Verte, Trois-Pistoles, Rimouski), ainsi que les Acadiens de la baie des Chaleurs (Bonaventure, Tracadièche (Carleton), Les Micmacs de Ristigouche, etc.).

\section{Le missionnaire et le professeur}

Dès son arrivée, La Brosse s'est fait ethnographe et linguiste. D'ailleurs il avait déjà à son crédit un dictionnaire en langue abénaquise.

La Brosse s'est intéressé à l'histoire des Montagnais. Pour établir son catalogue officiel des Montagnais en 1766, il a dû fouiller les registres antérieurs.

Il a également appris une foule de renseignements dans les manuscrits montagnais composés par ses prédécesseurs. En étudiant le contenu des catéchismes, des dictionnaires, des sermons ou exhortations, etc., il a connu toute l'histoire de la pénétration du christianisme chez eux. Il a dressé la liste de tous les missionnaires des Montagnais depuis 1640 sous le titre de Gentis Montanae in Deo Patres ac Pastores, qu'il insère dans le Troisième registre de Tadoussac (fol. 178v). Lui-même s'intitule le $21^{\mathrm{e}}$ missionnaire des Montagnais.

Ce contrat avec les prédécesseurs par l'intermédiaire de leurs écrits, joint à l'expérience déjà acquise chez les Abénaquis et les Malécites de la rivière Saint-Jean (1755-1756) et chez les Abénaquis de Saint-François (1758-1760), ajoutée à l'expérience de ses confrères, va lui dicter ses méthodes d'apostolat.

LA VISITE DES POSTES. - Le P. de La Brosse visite tous les postes à partir du printemps: Tadoussac, d'abord, à cause de son importance, puis les Îlets de Jérémie, Sept-Îles et parfois Portneuf. Au retour, il fait

8 Lettre de Mgr Briand à Saint-Luc Lacorne, Québec, 27 septembre 1768 (Arch. de l'évêché de Joliette). 
escale à Tadoussac et visite Chicoutimi. Entre la fête de sainte Anne (26 juillet) et le début de l'automne, il traverse le Saint-Laurent et visite les villages de Cacouna, l'Isle-Verte, Trois-Pistoles, Rimouski et la baie des Chaleurs (ce dernier endroit en 1771-1773).

Pour la visite des Postes du Roi, il utilise la barque ou la goélette de la compagnie de traite, qui le transporte gratuitement. Pendant l'hiver, il a recours aux raquettes, selon l'habitude des missionnaires du temps et probablement aussi du traîneau à chiens (voir conclusion: chanson composée par le P. de La Brosse sur son chien Jupiter).

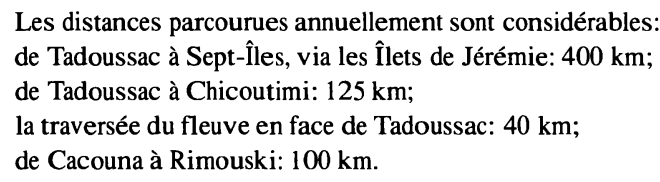

Les distances parcourues annuellement sont considérables: de Tadoussac à Sept-Îles, via les Îlets de Jérémie: 400 km; de Tadoussac à Chicoutimi: $125 \mathrm{~km}$; la traversée du fleuve en face de Tadoussac: $40 \mathrm{~km}$; de Cacouna à Rimouski: $100 \mathrm{~km}$.

La visite complète des postes exigeait un parcours approximatif de $1330 \mathrm{~km}$. Si on ajoute le voyage annuel à Québec, on obtient un trajet minimum annuel de $1730 \mathrm{~km}$ (ou 1030 milles). Pour l'époque, c'est considérable «autant de chemin que saint François-Xavier ${ }^{9}$ »?

LE RASSEMBLEMENT DU PRINTEMPS. - Avec le retour de la chasse, c'est le rassemblement du printemps. En plus des habitués de chacun des Postes, il y a presque toujours quelques nouveaux venus, qui viennent de l'intérieur, et qui ne sont pas chrétiens. D'après les registres, il en vient de Betsiamites, de Bon-Désir, de Chomonchouan, des Escoumains, de Manicouagan, de Mingan, du lac Mistassini, de la rivière Onistagane, de la rivière Noire, de la Pointe des Monts, des «Terres». Parfois leur origine transparait dans leur nom: Bersiamiskue (femme de Betsiamites) ou Agamiskue (femme du Lac).

$\mathrm{Au}$ début, c'est l'aspect commercial qui domine. Le nombre des employés du poste se fait plus nombreux pour accomoder les Montagnais qui viennent négocier leurs «peaux». Souvent ils n'auront pas la quantité de fourrures suffisante pour acquitter les dettes contractées l'automne précédent; car on leur a crédité alors leur approvisionnement en nourriture et en armes en vue de l'hivernement.

Pendant l'hiver, ils ont vécu en petits groupes de familles en forêt. La vie de société reprend maintenant sous l'autorité des chefs. Ils trouveront au poste la chapelle et le missionnaire qui baptisera leurs enfants, bénira les mariages, fera faire les Pâques à tout le monde, les fera prier pour leurs parents défunts inhumés dans le cimetière de la mission et aussi pour ceux qui sont morts en forêt au cours de l'hiver.

9 «Lettre circulaire de Monseigneur Paré», Saguenayensia, vol. 20, n 3-4, (mai-août 1978), p. $97-98$. 
À travers les registres, on voit apparaître une certaine hiérarchie du monde montagnais. Les chefs et leurs femmes sont souvent dans les «honneurs», comme parrains et marraines aux baptêmes, ou comme témoins aux mariages. Par exemple, les Annales de 1767 soulignent que le chef Pituabnu a été le premier à communier dans la nouvelle chapelle des îlets de Jérémie ${ }^{10}$.

Les cheferesses de prières jouent également un rôle important: gardiennes des chapelles, elles président les offices religieux en l'absence du missionnaire; et en tant que sacristines et catéchistes, elles l'assistent, quand il est au poste; leur influence est considérable.

Pendant le temps de la traite, la vie religieuse est intense: en quelques semaines, le missionnaire doit accomplir un ministère que les curés de paroisse répartissent sur une année. La plupart des Montagnais ne voient le missionnaire que quelques semaines par année; en plus des offices religieux ordinaires: messes, sermons, vêpres, prières du soir, etc., il y a l'administration des baptêmes, les confessions pascales, les mariages, l'enseignement du catéchisme et des prières en vue de la première communion. De plus, la célébration de fêtes, comme la sainte Anne, 26 juillet, prend un relief spécial à Tadoussac.

L'ENSEIGNEMENT DE LA BROSSE. - Ce qui caractérise l'enseignement religieux de La Brosse, c'est qu'il fonde son apostolat sur la connaissance de la lecture et de l'écriture; c'est donc une sorte de «religion du livre». Avant lui, les Montagnais n'ont pas disposé d'imprimés dans leur langue. Pour La Brosse, la connaissance de la lecture était indispensable à l'approfondissement de la foi et surtout à l'enseignement mutuel du catéchisme. C'est pourquoi, aussitôt son Catalogue des Indiens terminé, il fait imprimer son Nehiro-Iriniu et son Alphabet montagnais.

Pour aider aux Montagnais à apprendre à lire et à écrire, il simplifie le système d'écriture montagnais utilisé par les missionnaires précédents. Il dira, dans sa grammaire: "Nous avons introduit une nouvelle orthographe plus brève et plus facile, surtout pour les Indiens, à qui nous avons montré à lire ${ }^{11}$.

L'enseignement de la lecture et de l'écriture lui tient à cœur et il y consacre beaucoup de son temps; c'est un des aspects de son travail qu'il souligne le plus souvent et avec le plus de plaisir dans ses Annales; il s'y était consacré dès 1766 .

«La même année (1766-1767), à Tadoussac, pendant l'hiver, je montrai à lire aux Amérindiens, c'était la première fois et ce ne fut pas sans

10 Ann. $\mathrm{n}^{\circ} 5$.

11 Montanicae linguae Elementa... p. 2, $\mathrm{n}^{\circ} 8$. 
succès ${ }^{12}$ ». Il continue cette œuvre d'alphabétisation en 1768,1769 et de 1771 à 1776 , et probablement jusqu'à la fin de sa vie ${ }^{13}$.

En 1768, il fait féliciter les Montagnais de leur succès par Mgr Briand dans son mandement: «[...]. Nous avons été flatté, dit l'évêque - le GRAND PRIANT - d'apprendre que plusieurs d'entre vous s'appliquent à lire, apprennent la doctrine chrétienne, se l'enseignent mutuellement, et suppléent par là à l'absence du Missionnaire, qui ne peut vous visiter que successivement.»

Ce qu'il y a de plus remarquable dans les méthodes du P. de La Brosse, c'est d'avoir formé chez les Montagnais des collaborateurs catéchistes suffisamment instruits et suffisamment nombreux pour transmettre à leurs enfants et à leurs compatriotes la connaissance de la lecture et du catéchisme. Le contact avec le missionnaire ne durait que quelques semaines chaque année. Pour que le christianisme puisse s'enraciner, il fallait que les Montagnais eux-mêmes prennent en main leur éducation religieuse.

À l'été 1768, La Brosse organise une école de lecture, d'écriture et de catéchèse à Sept-îles; ce poste n'a pas reçu la visite du misisonnaire depuis six ans ${ }^{14}$. À l'Isle-Verte, pendant l'hiver 1774-1775, il utilise des copistes indiens pour transcrire des extraits de la Bible, qu'il a traduits en montagnais 15 .

L'ESSENTIEL DE LA FOI. - Le Nehiro-Iriniu (catéchisme et livre de prières) contient la doctrine enseignée par La Brosse aux Montagnais. Dans la transmission de la foi, peut-on dire que les Jésuites ont acculturé le message chrétien? En ce qui concerne les Montagnais, le Nehiro-Iriniu marque un effort pédagogique considérable pour l'enseignement chrétien à la mentalité montagnaise.

Les 150 questions et réponses sont simples et l'exposé est concret. Dieu s'appelle Tshiche Manitu (le grand Dieu), le Saint-Esprit: Miru Manitu (le beau Dieu); le démon: Mara Manitu (le mauvais Dieu).

La présentation du mystère de la Sainte-Trinité posait des problèmes aux missionnaires. L'unité de la Trinité est présentée ainsi: «Parce qu'ils approuvent toujours et qu'ils sont aimables l'un pour l'autre, le Père et le Fils, c'est pour cela qu'ensemble, avec le Saint-Esprit, ils ne font qu'un» $\left(\mathrm{n}^{\circ} 32\right)^{16}$.

\footnotetext{
12 Ann. $n^{\circ} 4$.

13 Ann. n ${ }^{\circ} 4,7,8,10,18,21,22,23$.

14 Ann. $\mathrm{n}^{\circ} 8$.

15 Ann. ${ }^{\circ} 21$.

16 Traduction littérale de M. Léonidas Larouche.
} 
On trouve dans le Nehiro-Iriniu des questions qui sont absentes des catéchismes français de l'époque et qui font état de préoccupations proprement montagnaises: le numéro 12 est formulé ainsi: «Est-ce qu'il mange, est-ce qu'il marche, le Grand Manitou?» Après la question sur la Trinité, on reprend le même sujet: «Des trois, quel est celui qui mange le plus, qui marche le plus, qui parle le plus (le mieux)» $\left(n^{\circ} 33\right)$. On pense, bien sûr, au nomadisme des Montagnais: il fallait être bon marcheur pour survivre dans la forêt pendant l'hiver; on pense aussi aux festins à tout manger (magushan), au cours desquels, on célébrait les heureux résultats de la chasse. Quant à la parole, le Grand Manitou des Montagnais se devait d'être un beau parleur; le terme montagnais qui signifie «parler» se traduit littéralement par «être plaisant à l'oreille ${ }^{17}$ ».

LA PRÉDICATION DU P. DE LA BROSSE. - Il existait des sermons du P. de La Brosse au temps du P. Charles Arnaud (1850-1859) ${ }^{18}$, mais ils sont perdus.

On peut quand même retrouver les principaux thèmes de sa prédication dans les catéchèses et les sermons des PP. Silvy et Coquart ${ }^{19}$, que La Brosse a abondamment annotés. Sous des titres plutôt rébarbatifs comme De Deo uno, De fine hominis, on enseignait l'histoire sainte, on traitait des sujets les plus divers, sur un ton familier. La Brosse s'en est certainement inspiré; de même, les Questionnaires du P. Pierre Laure pour les confessions ont vraisemblement orienté son enseignement.

On n'a peu de chance de se tromper en risquant d'autres thèmes comme l'ivrognerie, la fidélité des époux, l'importance de l'instruction religieuse qui sont exposés dans le Mandement de Mgr Briand de 1768 et dont le texte montagnais est du P. de La Brosse.

Il convient de noter ici qu'au sujet de l'ivrognerie et de l'impureté, le texte montagnais du Mandement de Mrr Briand est moins sévère que la version française officielle: dans celle-ci on lit: «Tout péché déplaît sans doute à Dieu, mais ces deux-ci endurcissent et aveuglent tellement le cœur de l'homme, que se convertissant rarement, il n'en obtient que difficilement le pardon de Dieu, tout prêt qu'il est à recevoir le pécheur pénitent». Le texte montagnais ajoute: «Néanmoins le Grand Manitou qui est trop bon pardonne toujours avec empressement à ceux qui ont pleinement satisfait et qui ont un vrai repentir ${ }^{20}$ ».

17 Traduction de M. Léonidas Larouche.

ī̄ Charles Arnaud, o.m.i., Journal (Arch. provinciales des Oblats, Maison provinciale, Montréal).

19 Manuscrits montagnais (Arch. l'archevêché de Québec).

20 Traduction de M. Léonidas Larouche. 
BAPTÊME ET CATÉCHUMÉNAT. - Presque tous les baptêmes sont des baptêmes d'enfants. De 1766 à 1782, La Brosse ne baptise que trois adultes. La chrétienté est ancienne. Il existe toutefois un catéchuménat exigeant et les conversions sont peu nombreuses.

La préparation au baptême dure au moins deux ans et, en certains cas, jusqu'à sept ans. Plusieurs mourront catéchumènes. Le P. de La Brosse semble avoir exigé une probation stricte et une instruction poussée. Pour être baptisé, il faut être «rectus instructus».

Les enfants ayant l'âge de raison, qu'ils soient de parents non chrétiens, ou catéchumènes, sont baptisés à condition de fournir des garanties suffisantes: savoir lire, écrire et connaître par cœur les prières et le catéchisme contenus dans le Nehiro-Iriniu. Les commentaires du P. de La Brosse sur sa pastorale des Montagnais sont extrêmement rares; mais il lui arrive parfois de marquer son admiration pour quelques montagnais particulièrement bien préparés au baptême. Ainsi il souligne le cas du jeune Martin Ukuiaban, fils de parents non chrétiens, mais très bien instruit par son parrain:

Baptisatur Martinus Ukuiaban catechumenus, duodecim annos natus, ex Kakashish et Katshbata, gentilibus, post duos probationis annos, catechismum calens et preces, et legere peritus instructus à suo patrino Thomas Uabstguanagan, [...].

A été baptisé Martin Ukuiaban, catéchumène, âgé de douze ans, fils de Kakashish et de Katshbata, non-chrétiens, après deux ans de probation; il connaît à fond son catéchisme et ses prières, sait lire parfaitement et a été instruit par son parrain Thomas Uabstguanagan [... $]^{21}$.

On peut en déduire que c'est la préparation qu'il considère comme idéale pour le baptême des adultes et aussi pour la première communion.

Le fait que les catéchumènes soient soumis à une assez longue probation et que sur 29 d'entre eux, seulement trois soient baptisés entre 1766 et 1782 (en supposant, bien sûr, que le registre soit complet), nous permet d'affirmer que la pression de la part du missionnaire et du milieu n'était pas très contraignante.

LE PARRAINAge ET LE TÉMOIgNAGE. - Contrairement à son prédécesseur le P. Coquart, La Brosse fait appel aux Montagnais euxmêmes pour remplir le rôle de parrains aux baptêmes et de témoins aux mariages des Montagnais. Même les enfants (métis) de Charles Taché, personnage important des postes, ont des parrains montagnais.

Il est évident que La Brosse a jugé les Montagnais plus aptes à remplir ces rôles et plus dignes de confiance que les Français des Postes. Ce

${ }^{21}$ Quatrième registre de Tadoussac, p. 25, L.-P. Hébert, p. 48. 
changement coïncide sans doute avec un niveau d'instruction religieuse plus poussé chez les chrétiens montagnais.

L'ENSEIGNEMENT DU CHANT ET DES CÉRÉMONIES. On sait que les Amérindiens avaient beaucoup de talent pour le chant religieux; des visiteurs comme Mrs Brookes qui a vécu au pays de 1764 à 1768 , l'ont signalé avec admiration 22 .

Les chants qu'on exécutait étaient d'abord les chants de la messe, comme le Kyrie, le Gloria, etc. en montagnais, mais aussi des chants traduits et adaptés du latin et du français, comme le Magnificat, l'O Salutaris Hostia, l'Ave verum, «Laissez paître vos bêtes», «Objets de ma nouvelle Flamme ${ }^{23}$ ».

En plus des cantiques inclus dans son Nehiro-Iriniu, le P. de La Brosse avait préparé des recueils manuscrits de cantiques: le P. Charles Arnaud les mentionne dans son journal de $1850^{24}$.

Dans ses Annales, il mentionne souvent qu'il a montré à chanter aux Montagnais ex modulatis notis. Faut-il traduire: «sur des airs connus»? comme d'ailleurs le faisait le P. Laure dans ses Prières et catéchisme? ou chanter à l'aide d'un texte noté? Cette dernière opinion est celle de James McKenzie, qui lors de son passage à Tadoussac en 1808 (26 ans après la mort du P. de La Brosse), écrit à propos des Montagnais: «They excel in singing hymns, and such as sing in church understand the notes which enable them to sing correctly ${ }^{25}$ ".

Il est évident que La Brosse a mis beaucoup de soin et de temps à la célébration des offices religieux, messes, vêpres, etc. Dans ses Annales de 1766-1767, il raconte:

Il n'y avait pas eu de vêpres en montagnais depuis de nombreuses années; je veillai à ce qu'on les chante selon le mode habituel, avec deux chœurs qui alternent; on les chanta pour la première fois le jour de l'Épiphanie 1767 [6 janvier] ${ }^{26}$.

Dans la petite chapelle de Tadoussac, le chant et la liturgie devaient revêtir un certain faste.

LA FÊTE DE SAINTE ANNE. - Ce n'était pas Noël, ni Pâques qui était le sommet de l'année liturgique, mais la Sainte-Anne. La Brosse

22 France Brookes, Histoire d'Émilie Montague, trad. de l'anglais par J. B. Robinest, Amsterdam, Changuion, Paris, Le Jay, 1770, vol. I, p. 25 (L'édition anglaise originale date de 1769).

23 P. Pierre Laure, Prières et catéchisme montagnais, p. 2, 9, 21, 36 (Arch. Deschâtelets, O.M.I., Ottawa).

24 Charles Arnaud, o.m.i., Journal, Arch. provinciales des Oblats, Maison provinciale, Montréal.

25 J. McKenzie, «The King's Posts», dans L. R. Masson, Les Bourgeois de la compagnie du Nord-ouest, Québec, A. Côté et Cie, 1890, II, p. 426.

26 Ann. $n^{\circ} 4$. 
la célèbrait habituellement à Tadoussac. Cette dévotion est très ancienne chez les Montagnais.

Très tôt ils ont été des habitués de Sainte-Anne de Beaupré, appelé autrefois Sainte-Anne du Petit Cap ou Sainte-Anne du Nord. Le cimetière de Sainte-Anne contient les corps de nombreux Indiens: entre 1670 et 1938, 74 d'entre eux y furent inhumés 27.

L'ancienne chapelle de Tadoussac était dédiée à Sainte-Croix. Lors de la reconstruction de 1747, par le P. Coquart, elle fut probablement dédiée à sainte Anne, selon la suggestion du P. Maurice ${ }^{28}$, bien qu'il n'en soit fait aucune mention dans les registres.

Au temps de La Brosse, en 1767, le «patronage» de sainte Anne est mentionné dans le Quatrième registre de Tadoussac, p. 22 et au début de son dictionnaire montagnais, p. 3.

Les registres indiquent la présence du P. de La Brosse à Tadoussac chaque année au moment de la sainte Anne (26 juillet). Sa célébration marquait le début de l'année. La Brosse l'appelait Festa Annalia, jouant ainsi sur les deux sens du mot latin annalia qui peut signifier «année» ou «Anne». Du reste, les calendriers indiens distribués aux Montangais chaque année répartissent l'année du 26 juillet au 25 juillet (fête de s. Jacques). Et le Nehiro-Iriniu, p. 40, contient un cantique à sainte Anne: TSHITSHITUA AN U-NIKAMUN.

L'HIVERNEMENT AU POSTE. - Au temps de La Brosse, les missionnaires ne suivent plus les Montagnais dans la forêt, comme l'avaient fait le P. Paul Le Jeune en 1633-1634 et le P. de Crespieul de 1671 à 1702. Pour hiverner, La Brosse choisit l'endroit où il se présente le plus grand nombre de familles.

Le choix se fait après entente avec les principaux agents de la compagnie de traite et la nouvelle est annoncée longtemps à l'avance. La Brosse hivernera plusieurs fois dans les Postes, à Tadoussac le plus souvent, et aussi aux Ilets de Jérémie. Quand le nombre de Montagnais n'est pas suffisant, ou que les besoins des Français l'exigent, il se transporte chez eux: Île d'Orléans (un hiver), Bonaventure (2 hivers), Isle-Verte et Rimouski (plusieurs hivers).

La présence du P. de La Brosse dans un poste donne à la vie religieuse une atmosphère d'étude. Tout le poste se transforme en école. C'est surtout pendant cette saison qu'il joue son rôle de professeur et que, comme

27 S. Baillargeon, Votre visite au sanctuaire de Sainte-Anne-de-Beaupré - Guide, 1978, p. 158-159; aussi dans Sainte-Anne-de-Beaupré, mai 1980, p. 208.

28 Troisième registre de Tadoussac, fol. 140r, L.-P. Hébert, p. 239-240. 
nous l'avons vu, il enseigne l'écriture, le chant, le catéchisme, les prières, les cérémonies religieuses, et qu'il prépare les enfants à la première communion.

C'est également le temps propice à la composition de ses ouvrages: catalogue des Indiens de Tadoussac, le Nehiro-Iriniu, l'abécédaire montagnais, les dictionnaires montagnais, la grammaire montagnaise. Que ce soit comme professeur, comme étudiant ou comme missionnaire, il montre une grande activité pendant les huit mois qu'il passe au poste.

LA VIE DES MONTAGNAIS EN FORÊT. - À l'automne, la plus grande partie des Montagnais partent pour la chasse; ils voyagent par petits groupes de familles sous la direction d'un responsable ou chef de chasse. Pendant l'hiver ils vivront des produits de leur chasse, du trappage, de la pêche et des provisions achetées au magasin du Poste. Ils accumuleront le plus grand nombre possible de peaux, puisque leur existence en dépend.

Pour observer les dimanches et les fêtes d'obligation, ils utilisent les calendriers qui leur sont distribués chaque été.

La vie en forêt n'était pas facile: le gibier n'est pas toujours suffisant et les Montagnais doivent parcourir de grandes distances pour s'approvisionner. Plusieurs meurent de faim et de froid en forêt pendant l'hiver. Le missionnaire porte leur nom au registre des décès au printemps, sur le témoignage des parents et amis.

Bien que le Quatrième registre de Tadoussac (1759-1784) ne soit pas à jour en ce qui concerne les décès (aucun après 1773), sur les 125 décès enregistrés de 1759 à 1773, on en compte 50 qui ont lieu en forêt. Parfois des familles complètes disparaissent pendant l'hiver ${ }^{29}$.

La mortalité infantile est élevée et les noyades nombreuses. Il arrive que le taux de mortalité s'élève subitement: pour la seule année 1769 , on compte 35 décès. Il y en aura 20 en 1770, année où la population montagnaise semble avoir été décimée par une épidémie ou la famine.

La mort en forêt était prévue dans le Nehiro-Iriniu et la question $\mathrm{n}^{\circ} 111$ était formulée de la façon suivante: «Que faire, quand on est en danger de mort en forêt, sans qu'on puisse recourir à un prêtre?» La réponse de onze lignes peut se résumer ainsi: on doit regretter ses péchés, par paroles, pensées et actions; dire «puissé-je ne pas mourir sans avoir reçu l'absolution du prêtre!»; réciter les prières, l'acte de contrition, etc., renoncer aux superstitions ${ }^{30}$.

29 Quatrième registre de Tadoussac, p. [8]sq, L.-P. Hébert, p. 11 sq.

30 Trad. de M. Léonidas Larouche. 


\section{Le linguiste}

Pour apprendre les langues amérindiennes, le P. de La Brosse suit la coutume des Jésuites: il utilise d'abord les dictionnaires composés par les prédécesseurs et compose ses propres ouvrages.

EN ABÉNAQUIS. - Deux ouvrages en abénaquis: un dictionnaire et un abécédaire abénaquis (Akitami...), imprimé.

1. Le dictionnaire abénaquis: Titre de la reliure: Radicum abanakae-

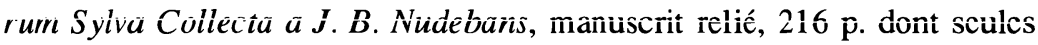
les dernières pages sont numérotées. Format 20 x 16,5 cm. Musée d'Odanak.

Le manuscrit comporte un dictionnaire latin-abénaquis (p. [1-116]) et un dictionnaire abénaquis-latin (p. [117-216]). Le titre d'origine se trouve à la page 117: Radicum Uâbanakaearum Sylva ex variis veterum recentiorumque manuscript. codicibus collecta \& Alphab. ordini restituta à $J$. B. Nudenans, anno 1760 (Dictionnaire des racines abénaquises compilé à partir de manuscrits et de recueils anciens et récents et disposé par ordre alphabétique par J. B. Nudenans, 1760).

L'auteur est bien le P. de La Brosse qui avait été missionnaire des Abénaquis de Saint-François de Sales de 1758 à 1760; tout le manuscrit est de son écriture.

2. L'Abécédaire abénaki: Akitami Kakikemesudi-Arenarag' Auikhigan, Messiui Arenâbak Uâbanakéuiak uitsi Pépâmkamigék éitsik, Kisittunésa Jan Baptist Nudenans, Mekazeusseuet Nege u-d-Aresigategui Patrihansa, Kebek - Dari, Arenarag'Auikhigebanik Broun, té Girmore, $1770,8,5 \times 14,5 \mathrm{~cm}, 7 \mathrm{p}$.

Traduction: Abécédaire à l'usage de tous les Abénaquis, quelque part qu'ils habitent. Fait par Jean-Baptiste Nudenans, jésuite, ancien missionnaire de Aresigategui (Rivière Saint-François), Québec, Brown et Gilmore, imprimeurs, $1770^{31}$.

Le P. de La Brosse en fit imprimer 600 exemplaires chez Brown et Gilmore en 1770 et les paya $£ 3 / 0 / 0^{32}$.

EN MONTAGNAIS. - Quatre ouvrages: un abécédaire, une grammaire, un dictionnaire et le Nehiro-Iriniu.

1. L'Abécédaire montagnais, Québec, Brown et Gilmore, 1767, 8p., $18 \times 28 \mathrm{~cm}$. Tirage: Trois mille exemplaires.

31 Traduction de MM. Gordon Day et Léonidas Larouche.

32 Arch. Nationales du Canada, Brown \& Gilmore Mss. MG 24, B 1, vol. 53. 
La page 8 comporte le colophon suivant: «Uabistiguiatsh, Massinahitsetuau, Broun gaie Girmore, 1767» (Québec [Cap Blanc], Brown et Gilmore imprimeurs, 176733).

Le nom de l'auteur n'est pas mentionné; mais La Brosse écrit dans ses Annales, $n^{\circ}$ 6: «Eodem anno [1767] in legentium et lecturorum gratiam alphabetha ter mille [...] typis excudendos curavi [...]». (La même année [1878], pour le bénéfice des Amérindiens qui savent lire et de ceux qui l'apprendront, je fis imprimer trois mille abécédaires [...]). De plus, Brown mentionne dans ses livres de compte qu'il a reçu un accompte de $£ 4 / 4 / 0^{34}$.

L'élève apprend d'abord les 16 lettres montagnaises, puis les lettres doubles sh, si, sk, st.; les syllabes; des exercices de lecture en syllabes détachées; on apprenait à lire sur des prières, selon l'habitude du temps. Cet abécédaire n'était sans doute qu'une adptation des abécédaires français de l'époque ${ }^{35}$.

2. La grammaire montagnaise. Manuscrit rédigé en latin, format $15 \times 17 \mathrm{~cm}, 201$ p., commencé aux Îlets de Jérémie en 1768, Archives Deschâtelets, O.M.I., Ottawa.

En-tête de l'ouvrage: Haec Montanicae linguae Elementa ex variis variorum Missionariorum Scriptis quae quidem vix reperiri potuerunt in unum corpus restituit Joannes Baptista de La Brosse Agesinas ex Societate Jesu Presbyter, qui fuit primus \& vigesimus Silvicolarum Montanorum, in Domini Regii Stationibus, in Deo Pater, \& Pastor.

Inceptum opus apud Insulas Jeremiae Papinachaeorum Stationem decimo Sexto Kalendas Decembres, anno à partu virginis octavo \& Sexagesimo post Septingent [esimum] \& millesimum.

Traduction de l'en-tête: «Ces éléments de langue montagnaise, élaborés à partir de divers écrits de divers missionnaires, - écrits qui n'ont pu être retrouvés qu'après beaucoup de recherches — ont été réunis en un seul corpus par Jean-Baptiste de La Brosse, originaire de l'Angoumois, Prêtre de la Compagnie de Jésus, qui fut en Dieu le vingt et unième Père et Pasteur des Montagnais dans les Postes du Domaine du Roi.

Ouvrage commencé aux Îlets de Jérémie, Poste des Papinachois, le 16 novembre 1768 de l'ère chrétienne.

33 Traduction deLéonidas Larouche.

34 Arch. Nationales du Canada, Brown \& Gilmore Mss, MG 24, B. 1, vol. 49.

${ }^{35}$ Le Grand Alphabei de 1800 utilise ia même méthode: Grand Alphabet divisé par syllabe pour instruire, avec grande facilité, les enfants à épeler, lire et chanter à l'église. contenant ce qui se chante à la Ste Messe, aux Vêpres et aux Complies. - À Québec, imprimé à la Nouvelle Imprimerie, rue des Jardins, 1800, 64p. in 16. 
Contenu. - Dans le premier chapitre de ses Montanicae linguae Elementa (De litteris), La Brosse présente l'état de la langue montagnaise à son arrivée à Tadoussac en 1766, et expose les adaptations qu'il a apportées à l'orthographe; ces changements sont importants, car ils ont eu une influence considérable sur la langue montagnaise; en effet, ils sont appliqués dans l'abécédaire et dans le Nehiro-Iriniu, deux ouvrages qui ont servi à l'apprentissage de la langue montagnaise pendant plusieurs générations.

Le P. de La Brosse avait d’abord observé que les écriis des anciens missionnaires n'étaient pas écrits selon la même orthographe et que la prononciation des syllabes et des mots n'était pas toujours la même. Pour faire disparaître toute ces ambiguittés, il propose une nouvelle orthographe:

Le nouvel alphabet proposé par La Brosse comprend 16 lettres, $a, b$, $\mathrm{e}, \mathrm{g}, \mathrm{h}, \mathrm{j}, \mathrm{i}, \mathrm{k}, \mathrm{m}, \mathrm{n}, \mathrm{o}, \mathrm{p}, \mathrm{r}, \mathrm{s}, \mathrm{t}$, u; les autres sont inutiles.

Les consonnes $b$ et $p$ se confondent, de même que g et $k$, $d$ et $t, j$ et sh.

Bien que les lettres $n, l$ et $r$ soient employées indistinctement (exemple: La Romaine, autrefois Ouraman et aujourd'hui Onaman), il garde le $r$ parce que cette lettre est typiquement montagnaise et d'un usage plus fréquent dans la prononciation.

La lettre u garde le son ou qui lui est propre; non pas celui que les Français sont les seuls à lui attribuer, mais celui qu'elle a chez les Anglais, les Allemands et tous les autres peuples.

Le u remplacera le 8 grec (l'omicron surmonté du upsilon), dont la plupart, dit-il, se servent mal à propos. Le 8 , en effet, est une diphtongue, u une voyelle simple. Jusqu'à maintenant on a publié beaucoup de livres et à l'avenir on devra en publier davantage; et comme les caractères grecs ne s'assortissent pas avec les caractères romains, à cause de la hauteur des lignes, il sera tout à fait difficile de faire imprimer des livres avec ce caractère. Plus l'impression et la lecture des livres seront simples et brèves, plus on devra les admettre.

La nouvelle orthographe que nous avons introduite est plus facile surtout pour les Amérindiens, à qui nous l'avons enseignée, et plus concise aussi.

Celui qui en fera usage arrivera facilement à se faire comprendre par toutes les tribus de la nation montagnaise, pusqu'en effet, il s'exprimera selon le génie authentique de la langue.

On peut suivre les progrès du P. de La Brosse dans l'apprentissage du montagnais dans le Quatrième registre de Tadoussac, qui contient la plupart de ses actes de baptêmes, mariages et sépultures. À son arrivée en 1766, il utilise la même orthographe que son prédécesseur, le P. Coquart, pour la transcription des noms montagnais: ch, et le signe 8 pour traduire le son ou. Mais à partir de 1767, il a systématisé sa nouvelle orthographe, à laquelle il restera toujours fidèle: le sh remplace le ch et le u traduit le 
son à la place du signe 8 ou de la diphtongue ou; ex. Iskueshish au lieu de Isk8echich.

3. Le dictionnaire montagnais-latin, manuscrit relié, 2 vol. sans pagination, 16,5 x 20,5. Commencé à Tadoussac en 1766. Archives Deschâtelets, O.M.I., Ottawa.

Traduction de l'en-tête de la page [3]: «Dictionnaire des racines montagnaises compilé à partir de divers écrits de divers missionnaires et classé par ordre alphabétique par Jean-Baptiste de La Brosse, prêtre de la Compagnie de Jésus, qui fut en Dieu le vingt et unième Père et Pasteur des Montagnais dans les Postes du Domaine du Roi».

«Ouvrage commencé à Sainte-Anne de Tadoussac le 8 novembre de l'année 1766 de l'ère chrétienne».

Total: 11791 mots.

4. Le Dictionnaire latin-montagnais, manuscrit relié, 2 vol., $16,5 \times 20,5 \mathrm{~cm}$. Commencé aux Îlets de Jérémie en 1772. Archives Deschâtelets, O.M.I., Ottawa.

En-tête de la page [3]: (voir plus haut).

«Ouvrage commencé aux Îlets-Jérémie, le 18 juin de l'année 1772 de l'ère chrétienne.»

Contenu linguistique. - Cet ouvrage constitue certainement l'étude la plus scientifique et la plus étendue de la langue montagnaise pour la deuxième moitié du $18^{\mathrm{e}}$ siècle. Souvent le $\mathrm{P}$. de La Brosse y fait des renvois à sa grammaire montagnaise. Vide Notas $N$. (voir Notes, numéro...), mais le numéro n'est pas complété. Il s'agit du dernier chapitre de sa grammaire montagnaise, constitué de Notae, numérotées de 1 à 55 (Montanicae linguae Elementa... p. 193-201).

Le P. de La Brosse consacra beaucoup de temps et beaucoup d'acharnement à la composition de ces deux ouvrages. Il nous en fait part dans ses Annales. En 1769-1770, il avait hiverné à Saint-Laurent, Île d'Orléans. «Je classai, dit-il par ordre alphabétique les mots du dictionnaire montagnais que j'avais commencé à compiler depuis trois ans; le travail fut considérable, le résultat plutôt modeste ${ }^{36}$ ». Quant à la partie latin-montagnais, commencée en 1772, il ne la termina que pendant l'hiver 1774-1775 à l'Isle-Verte: «Je mis la dernière main au dictionnaire que je préparais depuis huit ans ${ }^{37}$ ».

\footnotetext{
36 Ann. $\mathrm{n}^{\circ} 11$.
}

37 Ann. $\mathrm{n}^{\circ} 21$. 
Les manuscrits du P. de La Brosse servirent aux missionnaires Oblats quant ils arrivèrent au Saguenay pour apprendre le montagnais. Le P. Flavien Durocher, entre autres.

On peut connaître les ouvrages qui ont servi au P. de La Brosse pour la composition des ouvrages. Ce sont les Animadversiones et les Annotationes.

L. P. de La Brosse a laissé des traces de son passage sur un très grand nombre de manuscrits amérindiens en langue abénaquise, algonquine et montagnaise. Il annote, corrige, fait des remarques et des observations et parfois malices en mentionnant toujours que ce n'est ni par méchanceté, ni par gloriole, qu'il le fait, mais pour le bénéfice des utilisateurs éventuels, si futuri sint.

Les dictionnaires montagnais des Pères Bonaventure Fabre (1695) Antoine Silvy (1678-1684) et Pierre Laure (1726) ont été abondamment exploités. Le dictionnaire du P. Laure, L'Apparat français-montagnais (que M. David Cooter vient de publier aux Presses de l'Université du Québec) comportait 868 pages. Au moins 411 pages comportent des remarques ou des corrections du P. de La Brosse. Les catéchèses et les sermons des Pères Antoine Silvy et Coquart, conservés aux Archives de l'Archevêché de Québec, sont aussi copieusement annotés et corrigés.

La Brosse avait le sens du ridicule et l'esprit mordant; rien ne lui échappe; son ardeur de néophyte dans l'étude du montagnais le rend intransigeant pour les écrits de ses devanciers et sa déformation de professeur lui fait éprouver un malin plaisir à corriger les fautes des autres.

Les objectifs des dictionnaires du P. de La Brosse semblent uniquement d'ordre linguistique, alors que L'apparat français-montagnais du P. Laure est plutôt un livre de pastorale: en plus du contenu lexicographique proprement dit, le P. Laure faisait de nombreuses remarques d'ordre grammatical, ethnographique et religieux et souvent donnait en latin des conseils d'ordre pastoral à ses successeurs éventuels. La Brosse sépare ces trois contenus; dans son dictionnaire, il s'en tient à la langue; pour la grammaire et la religion il renvoie à ses Montanicae linguae Elementa et à son Liber superstitionum. (voir plus bas).

Mais pour avoir accès à ses dictionnaires, il faut recourir au latin, car c'est dans cette langue que La Brosse a rédigé plusieurs de ses ouvrages.

5. Le Nehiro-Iriniu n'a rien d'original en ce qui concerne le contenu, puisqu'il n'est que la reprise du catéchisme du P. Pierre Laure, datant de $17288^{38}$.

38 Communication de M. Léonidas Larouche. 
Le texte du P. Pierre Laure était le résultat d'une longue élaboration et d'une longue expérience dans l'enseignement de la religion aux Montagnais. Il y avait eu auparavant les essais des Pères Paul Le Jeune en 1633, Henri Nouvel de 1662 à 1669, Jacques Vaultier en 1676, François de Crespieul de 1671 à 1703, Louis André de 1690 à 1700 et Antoine Silvy de 1678 à 169939 .

La Brosse a fait un choix de prières et de chants dans la banque accumulée par ses prédécesseurs.

Cet ouvrage tiré à 2000 exemplaires $^{40}$ fut payé par les bourgeois de la compagnie de traite de Tadoussac ${ }^{41}$.

6. Traduction d' extraits de la Bible (perdue).

On ne connaît cet ouvrage perdu que par les Annales, $\mathrm{n}^{\circ}$ 21: [Hiver 1774-04-1775, à L'Isle-Verte]. «Je m'appliquai à la traduction de la bible en montagnais et comme mes ressources ne me permettaient pas de faire imprimer cet ouvrage, $j$ 'en distribuai des copies pour les faire transcrire par les Amérindiens».

L'ARCHIVISTE. - La Brosse avait une mentalité d'archiviste et le goût des vieux papiers: en vue d'apprendre la langue, il a recherché les manuscrits de ses prédécesseurs, il les a identifiés, classifiés et déposés dans des endroits sûrs.

Il indique ses sources: dans l'en-tête de son dictionnaire montagnais, il écrit qu'il a utilisé les matériaux tirés de divers manuscrits provenant de divers missionnaires. Au début de sa grammaire montagnaise, p. 1, il est plus explicite: il a élaboré ses Montanae linguae Elementa à partir de divers écrits de divers missionnaires, «écrits qui n'ont pu être retrouvés qu'après beaucoup de recherches».

Il y avait à Tadoussac (dans la sacristie de la chapelle, sans doute) un fonds de manuscrits, qu'il a certainement enrichi en y apportant tous les dictionnaires, grammaires, catéchismes, prières et sermons, qu'il a pu trouver à Québec et dans les chapelles des Postes du Domaine du Roi, en vue de son étude de la langue montagnaise. C'est lui-même qui désigne ce

39 Pour le contenu de cet ouvrage, voir L.-P. Hébert, «Le Nehiro-Iriniu du Père de La Brosse ou "L'influence d'un livre"” dans Une inconnue de l'Histoire de la culture - La production des catéchismes en Amérique française, sous la direction de Raymond Brodeur et de Jean-Paul Rouleau, Édition Anne Sigier, 1986, p. 57-79.

40 Ann. $n^{\circ} 6$.

41 Dans une lettre du 9 octobre 1782, adressée au Révérend William Morice, secrétaire de la Society for the Propagation of the Gospel (SPG), le colonel Daniel Claus reproche au principal actionnaire de cette compagnie, qui n'cst ricn moins que conseiller privé, etc. et qui se déclare membre de l'Église d'Angleterre, d'avoir fait imprimer à ses frais le livre de prières du P. de La Brosse. (Arch. Nationales du Québec, section anglicane). Il s'agit de William Grant (Dictionnaire biographique du Canada, V, p. 405). 
dépôt sous le terme d'archives (archio). En effet, il avait écrit en latin, sur le dictionnaire algonquin du P. Louis André, p. 2:

Le recueil suivant est de la plume du P. Louis André, qui fut missionnaire des Montagnais jusqu'en 1693. Il existe d'autres manuscrits du même missionnaire: son catéchisme, ses rudiments et sermons sont conservés aux archives de Tadoussac, sous le numéro...42

Le soin qu'il met à recueillir les anciens manuscrits lui fait regretter la disparition d'éléments importants du patrimoine des anciens jésuites: au débui de sà grammaire, ii écrivait:

«Il ne reste pas beaucoup d'écrits des anciens missionnaires, qui pourtant y avaient dépensé une somme considérable de travail. Certains ont été détruits lors de l'incendie de Chicoutimi en 1699, d'autres ont disparu à cause de la négligence des commis pendant les quinze ans que la mission a été privée de pasteur».

Et il poursuit: «Ceux qui restent, nous les avons rassemblés et nous les conservons avec soin; nous les avons utilisés soit pour élaborer cette grammaire, soit pour composer les lexiques ${ }^{43}$ ».

Toutefois, il voit ses archives amérindiennes d'une façon pratique et son respect des vieux manuscrits ne va pas jusqu'à l'empêcher de les annoter et de les corriger, pour le bénéfice de ceux qui les étudieront après lui.

LE LATINISTE. - Les ouvrages de La Brosse rédigés en latin sont les registres des Postes du Roi (une partie du Troisième registre de Tadoussac et tous les actes inscrits par lui au Quatrième registre de Tadoussac; les Annales Missionis... (Troisième registre de Tadoussac), le Liber superstitionum (perdu), son cours de philosophie (Cursus philosophicus datus a Patre Labrosse...), ses dictionnaires abénaquis et montagnais, sa grammaire montagnaise, et ses animadversiones et annotationes.

Pourquoi cet usage du latin? Sans doute ses ouvrages étaient-ils destinés à des prêtres? Mais les Pères Bonaventure Favre, Antoine Silvy et Pierre Laure n'avaient-ils pas rédigé leur dictionnaire en français? Voulaitil faire différent des ouvrages précédents? Des manies d'ancien professeur de latin?

42 Traduction du latin. Manuscrit conservé aux Archives de la Compagnie de Jésus à Saint-Jérôme, Qc.

43 Montanicae linguae Elementa... p. 2, $\mathrm{n}^{\circ} 7,8$. 
Il n'y a aucun doute qu'il est à l'aise en latin comme l'illustre son utilisation du Calendrier romain ancien ${ }^{44}$.

\section{L'ethnographe}

LES REGISTRES. - La Brosse a fait des actes dans un grand nombre de registres: Acadie (1755-1756), Hôpital-Général de Québec (1758), Saint-François-de-Sales (Abénaquis) (1758-1760), Saint-Françoisdu-Lac (1759), Saint-Michel-de-Yamaska (1758-1759), Saint-Louisde-Terrebonne (1760-1761), Saint-Henri-de-Mascouche (1750-1766), Saint-Charles-de-Lachenaie (1765), L'Isle-Verte (1766-1780), TroisPistoles (1770-1781), Saint-Germain de Rimouski (1771-1780), Ristigouche (1771), Bonaventure (1771-1773), Saint-Laurent de l'île d'Orléans (1769-1770), Saint-Louis de l'île aux Coudres (1766-1767), Éboulements (1766-1767), Petite Rivière Saint-François (1779) et deux registres des Postes du Domaine du Roi: le Troisième registre de Tadoussac, dans lequel il a inscrit ses Annales et surtout le Quatrième registre de Tadoussac qui contient ses actes de 1766 à 1782.

La Brosse émettait des certificats de baptême et de première communion, dont plusieurs sur des formulaires imprimés ad hoc; plusieurs de ses certificats ont été insérés dans les registres du Bas-Saint-Laurent.

Les registres tenus par La Brosse contiennent des éléments ethnographiques intéressants. Le Quatrième registre de Tadoussac nous révèle tout un monde: toponymie, géographie, état de la chrétienté, vie dans les Postes, vie en forêt, les divers groupes de la nation montagnaise, les structures de la société et le rôle des chefs.

À partir des données du Second registre de Tadoussac, édité par M. Léonidas Larouche, en 1972, le géographe Majella J. Gauthier, de l'Université du Québec à Chicoutimi, avait dressé une carte géographique du Domaine du Roi. Cette carte s'est enrichie grâce à l'apport du Troisième registre de Tadoussac, paru en 1976. Le Quatrième registre de Tadoussac, publié en 1982, a apporté du matériel toponymique nouveau. Les données de ce registre, provenant en grande partie du P. de La Brosse, ont permis d'établir une nouvelle carte du monde montagnais pour les années 1766-1782.

44 La Brosse date son arrivée à Tadoussac de: «quinto Idus Quintiles»: Quintiles: n'est pas le mois de mai, mais bien le cinquième mois du calendrier romain ancien, c'est-à-dire juillet; or comme les Ides de juillet tombent le 15 juillet; il faut compter 5 jours avant le 15 , en incluaunt le jour du départ et le jour de l'arrivée: donc le 11 juillet.

De même, il date le commencement de son dictionnaire montagnais de: «decimo sexto Kalendas Decembres»: il faut traduire le 16e jour avant les Calendes de décembre, c'est-àdire le 16 novembre. 
CATALOGUE DES INDIENS DE TADOUSSAC. - $\grave{A}$ son arrivée à Tadoussac en 1766, son premier geste avait été de faire le recensement des Montagnais chrétiens. Le texte original a été perdu, mais comme ce catalogue a été continué par l'abbé Jean-Joseph Roy, directeur du Séminaire de Québec, qui a fait la mission à Tadoussac de 1785 à 1795, on peut en avoir une bonne idée: chaque Montagnais est numéroté, avec indication du nom des parents, lieu et date de naissance. Le but était d'éviter à l'avenir de donner le même nom indien ou le même prénom chrétien à plusieurs personnes. Aussi de mieux voir la parenté et les empêchements de mariage, ce qui n'était pas facile chez les Montagnais. Le P. Pierre Laure dans son Apparat français montagnais en avait l'expérience ${ }^{45}$.

\section{LE CONTENU ETHNOGRAPHIQUE DE LA GRAMMAIRE} MONTAGNAISE. - Le P. de La Brosse avait de l'admiration pour la langue montagnaise. Au début de son manuscrit, il écrit:

On ne s'étonnera pas des variantes de la prononciation chez ces nations amérindiennes, qui errant continuellement à travers les forêts, ne peuvent pas facilement, à cause de l'éparpillement de la population, maintenir l'uniformité de prononciation. Si en effet chez les nations et les royaumes d'Europe, on trouve d'innombrables dialectes dans une même langue, à plus forte raison chez nos Montagnais. On devrait plutôt s'étonner (mirandum est) qu'après tant de siècles de nomadisme dans des lieux éloignés, ils puissent encore parler la même langue et se comprendre entre eux ${ }^{46}$.

\section{LE CONTENU ETHNOGRAPHIQUE DU DICTIONNAIRE} MONTAGNAIS. - Tout l'univers montagnais apparaît à travers le vocabulaire de la religion, de la géographie, de la chasse, de la forêt; on découvre la vie familiale, la vie sociale, le mode de vie, la psychologie et la mentalité des Montagnais, et d'une façon générale l'état de la culture montagnaise de la seconde moitié du XVIII' siècle.

LE LIBER SUPERSTITIONUM 47 - Nous ne connaissons cet ouvrage, malheureusement perdu, que par les vingt-quatre références que La Brosse y fait dans son dictionnaire montagnais.

Ce traité était subdivisé en numéros et contenait l'essentiel des croyances montagnaises. Le contenu était le suivant:

I. La Mythologie: mythes de Mishabush et de Tshakabesh.

II. Croyances et pratiques diverses: âmes, esprits, fantômes, les morts, les génies des eaux, etc.; évocation des génies; usage du tabac, festins à tout manger; jeter quelque chose au feu.

45 David Coooter, Apparat français-Montagnais [de P. Pierre Laure], Les Presses de l'Université du Québec, 1988, p. 585.

46 Montanicae linguae Elementa..., p. 3, $\mathrm{n}^{\circ} 10$.

47 Selon l'usage du temps, par exemple: P.-F.-X. Charlevoix, Histoire et description de la Nouvelle-France, Religion désigne le Catholicisme; l'Hérésie: le Protestantisme; Superstitions: la religion des Amérindiens. 
III. Le chaman et la magie: La cabane agitée; la cabane à suerie; le chant, l'enchantement du castor, du porc-épic, de l'ours, du caribou, de l'orignal; la danse, danse du feu; le jeûne; les songes, voir le gibier pendant le sommeil, utilisation des rêves; la pensée: voir à distance, prévoir l'avenir, détourner le mal par la pensée, penser en dormant; la parole: commander au vent, apaiser, détourner la tempête, l'orage; obtenir du gibier, rendre le chasseur heureux (felicem reddere venatorem), tuer.

IV. La divination: par le feu, la pyromancie, par le tremblement ou frémissement des paupières, des narines, etc.

V. La médecine: tente à suerie, médicaments et remèdes, etc.

Enfin il ne faudrait pas omettre le Mandement de Mgr Briand de 1768 (dont le contenu est du P. de La Brosse). Il fait connaitre les attitudes religieuses des Montagnais, les relations entre Européens et Montagnais, la question des mariages entre Blancs et Montagnaises, et ses conséquences sur le métissage, le problème de l'eau-de-vie.

LES CALENDRIERS. - Les Relations des Jésuites montrent que les calendriers indiens étaient en usage à Sillery dès 1640. La Brosse n'est donc pas l'inventeur, mais il les a répandus grâce à l'imprimerie.

Le P. de La Brosse a fait une grande consommation de ces calendriers: 1000 exemplaires en 1766, 100 exemplaires en 1768; une centaine d'exemplaires en 1769, 1770, 1771, 1772; 127 exemplaires par année de 1773 à 1778; et en 1778, il en commande pour sept années consécutives de 1779 à 1785, à raison de 500 ex. par année. Les livres de comptes des imprimeurs Brown et Gilmore les facturent au compte du P. de La Brosse 48 .

Un feuillet du calendrier de 1775-1776, (trouvé à la bibliothèque nationale du Québec à Montréal dans un exemplaire du Nehiro-Iriniu), révèle partiellement ce qu'étaient les calendriers du P. de La Brosse. La première colonne comporte le nom des mois ou des lunes et des fêtes mobiles; la deuxième le sanctoral: la fête de sainte Anne (26 juillet) jusqu'à la fête de saint Jacques ( 25 juillet).

\section{CALENDRIER MONTAGNAIS DU P. DE LA BROSSE}

(Traduction de M. Léonidas Larouche)

[Première colonne]
Akutshinu pihissimu:
Nouvelle lune
Uskagutshi pihissimu:
Premier quartier
Uauiasiu pihissimu:
Pleine lune (elle est ronde)

48 Arch. Nationales du Canada, Brown et Gilmore Mss, MG 24, B 1, vol. 49 à 125. 
Anittauiu pihissimu:

Uabikuni pihissimu:

Nitauatisheu pihissimu:

Uashtisiu pihissimu:

Pinastiu pihissimu:

Takulutateshịı nihissimu:

Usaku pihissimu:

Papiuatu pihissimu:

Epishinanamaskue pihissimu:

Uinasku pihissimu:

Nittshi pihissimu:

Uashikusamu pihissimu:

Pineaueu pihissimu:

+ Manatshitagan

O Papamutteuin

P Patshitatagan

T Tshigushimuin

\section{A Pikuutunaniu}

B Tshitapakuan

E Tshipaiatiku manatshihaganiu

G Apitshipaiatisu Jesus

H Uaskutsh upinihitisu Jesus

K Miru Manitu

N Tshitshitua Hostiuin
Dernier quartier

Lune des fleurs

juillet

Lune d'août (où les petits loups-marins croissent)

août

Lune où les feuilles jaunissent

septembre

Lune où les feuilles tombent

octobre

Lune d'automne (les jours

raccourcissent)

novembre

décembre

Lune de janvier

janvier

Lune de février

février

Lune où les marmottes sortent de leur tanière

mars

Lune où les outardes reviennent

avril

Lune où les arbres reverdissent

mai

Lune de juin (où les oiseaux pondent leurs oeufs)

juin

Dimanche ou fête d'obligation

Jour de procession

Abstinence

Jeûne

Mercredi des Cendres (on en met sur la tête)

Dimanche des Rameaux

Vendredi saint (on vénère la Croix)

Pâques (Jésus est ressuscité)

Ascension

(Jésus s'est élevé au ciel)

Pentecôte

(Le bon Manitou, le Saint-Esprit)

Fête-Dieu (sainte Hostie)

Il devait comporter six autres feuillets, de façon à contenir les douze mois de l'année (de juillet 1775 à juin 1776). 
Ce calendrier est bien adapté au cycle de la vie montagnaise: il s'étend de la fête de sainte Anne (26 juillet) à celle de saint Jacques (25 juillet); il était distribué au cours de l'été, avant le départ pour la chasse. $\mathrm{Ce}$ calendrier est simplifié et comporte moins de fêtes religieuses que celui du diocèse de Québec; leur nombre avait été diminué bien avant la campagne entreprise par Mgr Bailly de Messain en $1790^{49}$. Ainsi les fêtes de la Trinité, de la Visitation, des rogations, de l'ange gardien, des saints Innocents, etc. n'apparaissent pas au calendrier montagnais. Par contre la fête de sainte Anne prend un grand relief et tient lieu de Premier jour de l'année, comme on l'a vu précédemment. On remarquera que le mois des fleurs des Montagnais n'est pas le mois de mai, mais bien le mois de juillet, ce qui est plus conforme avec notre climat canadien.

LES ANNALES. - En qualité d'annaliste, La Brosse continue une tradition observée par les Pères de Crespieul, Laure, Maurice et Coquart, qui écrivent le compte rendu de leurs allées et venues annuelles et tenaient aussi la liste des bienfaiteurs de la Mission de Tadoussac. C'était la coutume chez les Jésuites; c'est à partir de ces comptes rendus que le Supérieur de Québec rédigeait les Relations des Jésuites au XVIIe siècle.

Deux documents composés par La Brosse doivent être rangés dans le genre «annales»: la Gentis Montanae in Deo Patres ac Pastores (liste des missionnaires des Montagnais insérée dans le Troisième registre de Tadoussac) et une longue lettre à Mgr Briand en date du 28 décembre 1771, dans laquelle il raconte son ministère auprès des Acadiens et des Micmacs de la Baie des Chaleurs.

\section{Résultats de l'œuvre du Père de La Brosse}

Peut-on évaluer l'œuvre de christianisation et d'alphabétisation du P. de La Brosse?

\section{La christianisation}

La source principale est le Quatrième registre de Tadoussac qui contient les actes de baptêmes et de mariages chez les Montagnais par le P. de La Brosse de 1766 à 1782. La compilation donne les résultats suivants:

49 Lettre à Mgr Hubert, 22 avril 1790, dans Mandement... des év. de Québec, Québec, A. Côté et Cie, 1858, II, p. 409-412. 


\begin{tabular}{lcc} 
& Baptêmes & Mariages \\
Tadoussac & 103 & 30 \\
Îlets-Jérémie & 76 & 10 \\
Sept-Îles & 65 & 2 \\
Chicoutimi & 13 & 4 \\
Portneuf & 7 & 0 \\
Isle-Verte & 2 & 0 \\
& & \\
\hline Total: & 266 & 46 \\
Moyenne annuelle: & 16,6 & 2,9
\end{tabular}

LES BAPTÊMES. - Comme on l'a vu plus haut, presque tous les baptêmes sont des baptêmes d'enfants.

LES MARIAGES. - Le nombre de mariages est relativement peu élevé. Il est probable que le Quatrième registre de Tadoussac ne tienne pas compte de tous les mariages; car on ne note pas de mariages pour les années 1780,1781 et 1782 . D'autre part, il y eut sans doute de nombreux mariages inscrits dans d'autres registres.

Enfin, il ne faut pas oublier que ce ne sont pas tous les Montagnais qui se marient devant l'Église.

ÉTAT DE LA CHRÉTIENTÉ EN 1782. - Selon Pierre Stuart, en 1778, la population totale des Montagnais du Domaine du Roi était de 3500 âmes ${ }^{50}$. Pour la population chrétienne, on a d'autres indices: ce sont les calendriers que La Brosse fait imprimer annuellement. De 1766 à 1773, la quantité varie de 100 à 127 exemplaires par année (excepté la première année: 1000 exemplaires). À partir de 1778, il commandait 500 exemplaires annuellement. En supposant que les familles montagnaises soient composées de cinq membres et que chacune reçoive un calendrier, on peut estimer la chrétienté montagnaise à 100 familles avant 1778 et cinq cents après cette date; donc une population chrétienne variant de 500 à 2500 âmes entre les années 1766 et 1778.

Dans l'évaluation de ces données, il faut tenir compte des progrès de la connaissance de la lecture, progrès qui pourraient expliquer l'usage d'un plus grand nombre de calendriers indépendamment de l'augmentation de la population chrétienne.

50 Journaux de la Chambre d'Assemblée du Bas-Canada, 1823-1824, vol. 33, app. (R). 
En attendant des études plus scientifiques, il vaut mieux s'en référer au Catalogus generalis... de l'abbé J.-J. Roy, successeur du P. de La Brosse, qui fixe à 1132 le nombre des Montagnais chrétiens pour la décennie 1785-179551.

\section{L'alphabétisation}

Du vivant même de La Brosse, ses efforts d'alphabétisation furent tangibles. Le Catalogus generalis... de l'abbé J.-J. Roy est un bon instrument d'évaluation de l'enseignement de La Brosse; sur les 1132 Montagnais énumérés, 106 noms ( 50 hommes et 56 femmes) sont notés au point de vue lecture et 122 ( 56 hommes et 66 femmes) au point de vue pratique religieuse.

Sauf deux, ils sont tous nés entre 1732 et 1778 . Ils ont pratiquement tous appris à lire et à écrire au temps de La Brosse.

\section{Résultats:}

Deux ont la note Optime (très bien) pour la lecture et la religion.

104 ont la note Bene (bien) en lecture et en religion;

Un (1) a la note Bene en lecture et Med [iocriter] (moyen) en religion.

9 (non notés) en lecture ont $M e d$ en religion;

Total: 106 notés en lecture;

122 notés en religion (dont 16 ne savent pas lire).

On remarquera que la note de lecture est presque toujours la même que celle de la religion et que parmi les sept noms notés Med. (moyen) en pratique religieuse, six ne savent pas lire. La corrélation entre la connaissance de la lecture et la pratique religieuse est donc très grande.

LES SIGNATURES INDIENNES. - L'absence de signatures dans les registres n'est pas toujours significative; on ne trouve aucune signature montagnaise dans les registres du P. de La Brosse.

Le registre D des Postes du Domaine du Roy (1785-1796), tenu par l'abbé J.-J. Roy, contient quelques signatures indiennes en 1785 et 1786; dans la suite la plupart des actes se terminent selon la formule de l'époque «ont déclaré ne savoir signer» ou «tous illettrés».

Or, dans plusieurs actes, les témoins qui sont dits «illettrés» savent signer en réalité. C'est le cas, entre autres, de Jean-Baptiste Assini, instruit par le P. de La Brosse et de plusieurs autres qui sont recensés dans le

51 Jean-Joseph Roy, Catalogus generalis totius Montanensium gentis, fol. 36v. (Arch. de l'archevêché de Québec). 
Catalogus generalis... de l'abbé J.-J. Roy comme sachant lire et écrire. La formule finale «ne savent signer» ne doit donc pas être prise à la lettre.

Dans les registres qui suivent, de 1799 à 1816, les signatures de Montagnais sont nombreuses: on en trouve en 1799, 1801, 1802, 1807, $1808,1809,1810,1815$, etc. Toujours en lettres moulées, selon l'habitude montagnaise, apparaissent les signatures de Pier Napesh (12 fois), JeanBaptiste Assini (11 fois), Ba Kapapeskueues (2 fois), Jak Tshiutesish (2 fois), Joseph Matauashuss, J. Tshishiuetshishi, Raui Kakamikus, J. B. Tshisherinish̆, Jer. Šhishenapeu, B. Esturu, Patir Assinı.

TÉMOIGNAGE DU XIXe SIÈCLE. - Au cours de leur visite annuelle, les successeurs de La Brosse au XIX ${ }^{\mathrm{e}}$ siècle ne consacreront pas beaucoup de temps à l'enseignement de la lecture, de l'écriture et du catéchisme. Ce sont les Montagnais eux-mêmes qui assureront cet enseignement. Avant l'arrivée des Missionnaires Oblats de Marie-Immaculée en 1844, la connaisance de la lecture se perpétue par l'enseignement mutuel, comme en font foi les témoignages de James McKenzie en 180852, d'Alexander Fraser en 182453 et de l'arpenteur Joseph Bouchette en $1828^{54}$.

De 1782 à 1844 , il y eut une quinzaine de missionnaires qui vinrent faire la mission annuelle; «mais, dit le P. Charles Arnaud, ne connaissant pas la langue, ils ne pouvaient faire tout le bien qu'ils désiraient ${ }^{55}$ ». La connaissance de l'écriture se maintient pourtant, car une requête des Montagnais de la Rivière Godbout, en date du 28 juillet 1845, contient les signatures de 18 montagnais ${ }^{56}$ et en 1847 , les montagnais présentent une pétition au Parlement du Canada Uni, signée par 127 d'entre eux, dont six chefs 57 .

52 «La plupart d'entre eux parlent, ou plutôt écorchent la langue française et lisent, écrivent et correspondent dans leur propre langue. Ils écrivent en lettres moulées. [...]. Ils excellent dans le chant des hymnes et ceux qui chantent à l'église comprennent les notes suffisamment pour chanter correctement. (J. McKenzie, «The King's Posts», dans L. R. Masson, Les Bourgeois de la compagnie du Nord-Ouest, Québec, Côté, 1890, II, p. 426).

53 «La nation des Montagnais ou Ichini [répandus] jusqu'au Lac Saint-Jean répètent [sic] leurs prières et lisent le catéchisme dans leur langue» (Journaux de la Chambre d'Assemblée du Bas-Canada, vol. 33, App. (R)).

54 «Un missionnaire catholique visite Chicoutimi deux fois l'année, et enseigne aux naturels les premiers principes de la religion, dont les Jésuites avaient composé en langue créenne [montagnaise] un catéchisme qui circule parmi eux» (Joseph Bouchette, Journal du parti-explorateur de la rivière St-Maurice (Journaux de la Chambre d'Assemblée du BasCanada, 1828).

55 Arnaud à Casgrain, 20 janvier 1899, Arch. provinciale de Oblats de MarieImmaculée, Montréal.

56 George Duberger, River Godbout 29 july 1845, Report of His Exploration (Arch. Nationales du Québec, QBC-13-19).

57 Arch. de la Chancellerie de l'Archevêché de Montréal 295.098/847-3A. 
Avec l'arrivée des Missionnaires Oblats de Marie-Immaculée en 1844, la tradition se continue: le P. Flavien Durocher écrit en 1846: «Les étrangers étaient étonnés de voir ces habitants des forêts le livre à la main pendant les offices, surtout en apprenant qu'ils se transmettaient de père en fils, sans le secours d'instituteurs étrangers, l'éducation primaire qu'ils avaient reçue des enfants de saint Igance ${ }^{58}$ ».

Les témoignages du P. Arnaud en $1850^{59}$, de l'abbé François Pilote en $1851^{60}$, de David Price en $1858^{61}$, de Joseph-Charles Taché en 186362 , du P. Zacharie Lacasse en $1876^{63}$ et du juge O'Brien en $1880^{64}$ vont tous dans le même sens.

58 F. Durocher, o.m.i., Lettre à l'archevêque de Québec, Tadoussac, 25 juillet 1846, dans Rapport sur les missions du diocèse de Québec, juillet 1847, $\mathrm{n}^{\circ}$ 7, p. 123.

59 Au sujet des Montagnais des Îlets de Jérémie: «Ces sauvages sont les plus instruits de la côte: ils savent tous lire et écrire» (Ch. Arnaud, o.m.i., Lettre... Baie des Ha! Ha!, 30 septembre 1850, dans Rapport sur les misisons du diocèse de Québec, mars 1851, $\mathrm{n}^{\circ} 9$, Québec Côté et Cie, 1851, p. 36).

En 1858, il complètera sa pensée: Chez les Montagnais, dit-il, il y a «autant d'écoles que de pères de famille, car chacun apprend à lire et à écrire à ses enfants" (Escoumains, 7 décembre 1857, dans Rapport des commissaires... 21 Victoria, Appendice $\left(\mathrm{n}^{\circ} 21\right)$, A. 1858, p. 184).

60 «Chose étonnante! après deux cents ans, les sauvages du Saguenay, malgré leur genre de vie nomade et vagabonde, savent encore tous lire. Chaque famille conserve avec soin son livre de prières et de cantiques, en fait largement usage les dimanches et fêtes que tous observent religieusement suivant un calendrier particulier qu'ils entendent à merveille. Plusieurs savent le plain-chant. Sous ce rapport beaucoup de familles parmi nous sont bien en arrière de ces pauvres sauvages. Notre siècle est pourtant bien fier de ses lumières! Le sauvage du Saguenay apprend à lire comme il apprend à tirer sur le gibier ou à conduire un canot. C'est qu'il regarde l'un comme aussi nécessaire que l'autre à sa double vie morale et physique». (François Pilote, Le Saguenay en 1851, Québec, A. Côté et Cie, 1852, p. 27-28).

61 «Ces sauvages ont tous embrassé le christianisme et sont membres de l'Église catholique romaine; ils sont très dévôts pour la plupart, et savent leurs prières et les hymnes dans leur propre langue. Les missionnaires qui travaillent à leur régénération spirituelle, ont porté toute leur attention sur ce point, et réduit leur dialecte en un système régulier, en publiant plusieurs livres dans leur langue; la plupart de ces sauvages savent lire et écrire». (Rapport des commissaires spéciaux, nommés pour s'enquérir des affaires des Sauvages en Canada, Toronto, 1858, (21 Victoria, Appendice $\left(\mathrm{n}^{\circ} 21\right)$, A. 1858), p. 36.

62 «Ce qu'il y a de bon, dit-il, et de beau chez les Montagnais, c'est que les enfants apprennent leurs prières, leur catéchisme et le chant même, sans que le missionnaire s'en mêle presque. Ils savent lire et écrire, et c'est dans la cabane que les premiers préceptes de la religion s'enseignent par le père et la mère». (J.-C. Taché, "Forestiers et voyageurs», dans Les soirées canadiennes, $3 \mathrm{e}$ année, 1863, p. 128).

63 «Votre première occupation devra être de montrer à lire à ces pauvres sauvages pour qu'ils puissent s'instruire eux-mêmes, le catéchisme occupera presque tout le temps». (P. Zacharie Lacasse, o.m.i., "Missions des Naskapis», lettre du Révérend P. Lacasse, o.m.i., Saint-Sauveur, $1^{\text {er }}$ avril 1878, p. 113).

64 A propos des Montagnais de Betsiamite. "Cet usage chez les "anciens" de la tribu d'apprendre à lire et à écrire aux enfants, date des premiers missionnaires jésuites. C'est une obligation sacrée à leurs yeux; aussi est-il rare de rencontrer un adulte montagnais qui ne sache lire et écrire». (François-H. O'Brien (magistrat de district), «Rapport spécial dc M. O'Brien, Québec, 18 octobre 1880", dans Documents de la session ( $\left.n^{\circ} 5\right) 44$ Victoria, A. 1880; Rapport du commissaire de l'instruction publique $\left(n^{\circ} 5\right)$ - Rapport des commissaires, p. 123. 
Quant à l'usage des calendriers montagnais, imprimés pour la première fois en 1766, par le $\mathrm{P}$. de La Brosse, il se maintient sous le P. Le Courtois, eudiste, qui desservit les Montagnais de 1799 à 1814; il en fit imprimer régulièrement en $1804,1805,1809,181065$ et on en faisait toujours usage en $1848-1849$, en 1851 et en 185866 .

\section{Conclusion}

Avant de conclure, il n'est pas sans intérêt de savoir ce que les supérieurs du P. de La Brosse pensaient de lui avant qu'il entreprenne son apostolat missionnaire.

Il existait chez les Jésuites des catalogues ou registres, dans lesquels les supérieurs jaugeaient leurs sujets: à quatre reprises, on a des appréciations de La Brosse: en 1743, 1749, 1754 et 1757. Au point de vue intellectuel (ingenium, judicium, prudentia), il est habituellement noté Optime; ses succès dans ses études ne font aucun doute. Ils sont hautement cotés et particulièrement en philosophie et en théologie.

Quant à son tempérament, il est qualifié de firma, optima, indolis, temperata, temperata non nihil audens ad biliosam: un caractère agréable, ferme, modéré et quelque peu enclin à la colère. Sa maturité mérite des éloges et particulièrement dans le domaine spirituel. Pour les aptitudes, il est apprécié ad omnia, apte à toutes fonctions, ce qui était un idéal dans la Compagnie de Jésus; sa santé est toujours qualifiée de solide. La Brosse était alors dans la trentaine.

Les prévisions étaient justes: La Brosse ne s'est pas démenti et cela, même après la suppression des Jésuites par Clément XIV en 1773.

Sa bonne santé se maintient jusqu'en 1775 , alors que, malade, il doit hiverner à Rimouski, malgré lui. Il meurt à 58 ans. Il ne restait plus que cinq jésuites vivants.

Il était capable de fermeté et de colère jusqu'à affronter les autorités religieuses ${ }^{67}$, mais aussi d'humour. Après plusieurs années d'effort pour composer son dictionnaire montagnais, il écrira dans ses Annales: «Labore multo, fructu forte modico" (beaucoup d'efforts, mais peut-être sans grand résultat).

65 Arch. Nationales du Canada, MG 24, B 1, 81.

66 Le Tsistekaigan... est imprimé chez Côté à Québec en 1848. Pour l'année 1851, voir le témoignage de l'abbé François Pilote, Le Saguenay en 1851, p. 27-28; pour 1858, le témoignage de David Price, Rapport des commissaires, (21 Victoriae, appendice $\left(\mathrm{n}^{\circ} 21\right)$, appendice 11, p. 181). Rimouski).

${ }^{67}$ Lettre au Grand Vicaire Gravé, Tadoussac, 21 avril 1780 (Arch. de l'archevêché de 
Il s'impatiente devant la négligence de style et les fautes de ses «révérends» prédécesseurs, il les corrige, pensant que cela pourrait être utile à ses successeurs, si futuri sint! (si jamais il y en a!). La teneur de ses corrections dénote certainement un talent particulier pour l'ironie et la caricature.

Le quatrain écrit contre le $\mathrm{P}$. Gélase de Lestage est célèbre. Ce Récollet avait commis de nombreuses fautes dans le Troisième registre de Tadoussac en 1716. En 1766, le P. de La Brosse écrit:

«Vere prius flores, aestu numerabis aristas,

Poma per autumnum, frigoribus nives;

Quam voces perperam exaratas ab illo Rev. Patre Gelasio de

Lestage contra omnes tum Grammaticae, tum Orthographiae leges 68 ».

Et ce quatrain - des «Facheux» relevé par J.-C. Taché. Pour se débarrasser des visiteurs importuns qui lui faisaient perdre son temps, au Poste de Chicoutimi, le P. de La Brosse aurait affiché à sa porte les vers suivants:

«Pour un homme occupé, rien de plus ennuyeux

Que de gens désœuvrés la visite importune;

J'aimerais presque autant qu'on me crevât les yeux,

Que de venir ici, pour m'en procurer une ${ }^{69}$ ".

À son passage à Tadoussac, James McKenzie a recueilli deux vers d'une chanson du P. de La Brosse, dédiée à son chien Jupiter:

He had also poetical talents, and composed a song upon favorite dog, which began thus:

Jupiter par la vieillesse

N'a pu de poils aux fesses 70 .

Après tous les efforts que La Brosse avait déployés pour enseigner le chant religieux aux Montagnais, il aurait été bien étonné d'apprendre que l'on ne retiendrait que ces deux vers de ses talents musicaux et poétiques!

Ces aspects plaisants de la personnalité du P. de La Brosse ne doivent pas nous faire oublier l'essentiel de son œuvre. Chez lui, le professeur est toujours présent avec ses manies, sans doute, mais aussi avec ses talents

68 Troisième registre de Tadoussac, fol. 19v, L.-P. Hébert, p. 51. Le sens général: «Il est plus facile de compter les fleurs au printemps, de calculer les épis de blé à l'été, de dénombrer les fruits à l'automne, de mesurer les neiges de l'hiver, que de relever les fautes commises par cet illustre Révérend Père Gélase de Lestage contre toutes les lois de la grammaire et de l'orthographe». Ce quatrain semble inspiré des Géorgiques I, de Virgile.

69 Lettre de J.-C. Taché au P. J. Tailhan, s.j., Québec, 9 janvier 1863, Arch. de la Compagnie de Jésus de France (Chantilly); copie microfilmée aux Arch. Nationales du Canada, boîte 16, article 4, Canada XVIIIe siècle, Bobine f-720; aussi dans Les Soirées canadiennes, $3^{\mathrm{e}}$ année, 1863, p. 121-122.

70 J. McKenzie, «Le King's Posts», dans R.-L. Masson, Les Bourgeois de la compagnie du Nord-Ouest, Québec, Côté et Cie, 1890, II, p. 412. 
extraordinaires d'éducateur de la foi. L'originalité de son œuvre est d'avoir montré à lire et à écrire aux Montagnais, d'avoir mis à leur disposition un abécédaire et un catéchisme, qui leur ont permis de se transmettre entre eux l'écriture et les connaissances religieuses. S'il est vrai que le bon professeur cherche à former des élèves qui se passeront de lui le plus tôt possible, La Brosse a atteint son but et ses talents de professeur ont bien servi le missionnaire, qu'il fut avant tout.

\section{Un saint et un héros?}

Sur la rive sud du Saint-Laurent, le P. de La Brosse s'est fait une réputation de thaumaturge. Lorsqu'en 1906, Mgr Albert Blais érigea la desserte de Rivière Trois-Pistoles, il la mit sous le patronage de saint JeanBaptiste, en l'honneur du P. de La Brosse:

[...] et nous sommes heureux de rappeler le vénérable père de La Brosse religieux de la Compagnie de Jésus, cet illustre et intrépide missionnaire qui a desservi avec tant de zèle, d'abnégation, de dévouement et d'édification les divers groupes de fidèles échelonnés sur les rives de cette partie du Saint-Laurent et à la vertu duquel la tradition attribue des effets merveilleux dus à son puissant crédit auprès de Dieu dans la paroisse même de Trois-Pistoles ${ }^{71}$.

Dans sa lettre circulaire du 3 janvier 1978, à l'occasion du centenaire du diocèse de Chicoutimi, Mgr Marius Paré, évêque de Chicoutimi rendait hommage aux missionnaires jésuites et en particulier:

[...] au Père Jean-Baptiste de La Brosse, vénéré comme un saint, qui en seize ans, même après la suppression de son ordre, en 1774, parcourut à la poursuite des âmes autant de chemin que saint François-Xavier 72 .

Mais le P. de La Brosse fut aussi héros de légendes: les deux plus connues sont la légende des Cloches et la conjuration du feu, représentée par un bronze du sculpteur Alfred Laliberté.

Pour que le personnage de La Brosse ait pu donner prise à la légende, il fallait que son magnétisme personnel fût puissant; cette condition étant remplie, le processus de la légende peut se mettre en marche; pour idéaliser son héros et en conserver la mémoire, la légende se permet toutes sortes de licences, entre autres la "déformation» de l'histoire; c'est le prix à payer pour devenir héros.

71 Archives de l'Archevêché de Rimouski.

72 Saguenayensia, vol. 20, nº 3-4, (mai-août 1978), p. 97-98. 


\section{Bibliographie complémentaire}

Chambre, Alexandre, Le R. P. J.-B. de La Brosse, né à Jauldes (Charente), mort à Tadoussac (Saguenay), [s.é., n.d.], [1904], XIX et 362 p.

Dragon, A., Trente robes noires au Saguenay, Chicoutimi, La Société historique du Saguenay, $\mathrm{n}^{\circ}$ 24, [1970], $397 \mathrm{p}$.

Rochemonteix, Camille de, s.j., Les Jésuites et la Nouvelle-France au XVIII ${ }^{e}$, Paris, A. Picard et fils, 1906, 2 vol.

Roy, J.-E., Au royaume du Saguenay - Voyage au pays de Tadoussac, Québec, Côté, 1888, 235 p.

Tremblay, Victor, Histoire du Saguenay depuis les origines jusqu'à 1870, Éd. nouvelle, Chicoutimi, La Société historique du Saguenay, $\mathrm{n}^{\circ} 21$, $1968,465 \mathrm{p}$. 